\title{
The $\mathrm{HBx}-\mathrm{CTTN}$ interaction promotes cell proliferation and migration of hepatocellular carcinoma via CREB1
}

\author{
Yajun $\mathrm{Li}^{1}$, Yongming $\mathrm{Fu}^{1}$, Xingwang $\mathrm{Hu}^{1}$, Lunquan Sun${ }^{2}$, Daolin Tang ${ }^{3}$, Ning Li ${ }^{4}$, Fang Peng ${ }^{5}$ and Xue-gong Fan ${ }^{1}$
}

\begin{abstract}
Hepatitis B virus-encoded X protein ( $\mathrm{HBX}$ ) acts as a tumor promoter during hepatocellular carcinoma (HCC) development, probably by regulating the expression of host proteins through protein-protein interaction. A proteomics approach was used to identify HBx-interacting proteins involved in HBx-induced hepatocarcinogenesis. We validated the proteomics findings by co-immunoprecipitation and confocal microscopy. We performed cell proliferation, migration assays and cell cycle analyses in HCC cells. Finally, we confirmed the clinical significance of our findings in samples from patients. We found that cortactin (CTTN) is a novel HBx-interacting protein, and HBx regulates the expression of CTTN in the HCC cell lines MHCC-LM3 and HepG2. Mechanistically, by upregulating the expression of CAMP response element-binding protein (CREB1) and its downstream targets, such as cyclin D1 and MMP-9, the effects of the $\mathrm{HBX}-\mathrm{CTTN}$ interaction on the enhancement of cellular proliferation and migration were maintained by inhibiting cell cycle arrest. In addition, we demonstrated that the levels of CTTN and CREB1 were closely correlated in clinical samples from HBV-infected patients with HCC. Overall, our data suggests that HBx contributes to cell migration and proliferation of HCC cells by interacting with CTTN and regulating the expression of CTTN and CREB1. Therefore, the $\mathrm{HBx} / \mathrm{CTTN} / \mathrm{CREB} 1$ axis is a potential novel therapeutic target in HCC.
\end{abstract}

\section{Introduction}

Hepatocellular carcinoma (HCC) is an aggressive human malignancy ${ }^{1-3}$. Hepatitis B virus (HBV) infection is the most common risk factor for HCC, and there are $\sim 250$ million chronic HBV carriers in the world ${ }^{4-7}$. Although HBV vaccines have obviously decreased the number of new HBV infection cases, they have limited benefits for previously diagnosed patients with chronic HBV infection ${ }^{8,9}$. The HBV genome comprises four overlapping open-reading frames: $\mathrm{preC} / \mathrm{C}$, preS/S, $\mathrm{P}$, and $\mathrm{X}^{10}$. The $\mathrm{X}$ protein $(\mathrm{HBx})$, encoded by the $X$ region, is a multifunctional viral regulator involved in viral

\footnotetext{
Correspondence: Fang Peng (pengfang@csu.edu.cn) or

Xue-gong Fan (xgfan@hotmail.com)

'Department of Infectious Diseases and Hunan Key Laboratory of Viral

Hepatitis, Xiangya Hospital, Central South University, Changsha, China

${ }^{2}$ Center for Molecular Medicine, Xiangya Hospital, Central South University,

Changsha, China

Full list of author information is available at the end of the article.

Edited by G. Giannelli
}

pathogenesis and carcinogenesis, and it plays an important role in HBV-related HCC processes, such as autophagy regulation, DNA repair inhibition, posttranscriptional regulation, and cell cycle arrest ${ }^{9,11-14}$.

HBx interacts with many host targets, and as such, it is important in viral hepatocarcinogenesis ${ }^{15-19}$. For example, $\mathrm{HBx}$ activates transcription by interacting with transcription factors or complexes, including P53, C/EBP, Sp1, and STAT3 ${ }^{20-27}$. HBx also activates various cellular signal transduction pathways related to its transactivation, such as the NF- $\mathrm{B}$, AMP-activated protein kinase (AMPK), and MEKK1/Jun kinase signaling pathways ${ }^{22,28}$. Thus, the identification of novel $\mathrm{HBx}$-host interactors and the pathways they are involved with might aid in the development of effective therapies for patients with HCC.

In this study, we looked for $\mathrm{HBx}$-interacting proteins in $\mathrm{HCC}$ and explored their mechanism of action. Our data demonstrated the physical association between $\mathrm{HBx}$ and CTTN and identified the new $\mathrm{HBx} / \mathrm{CTTN} / \mathrm{CREB} 1$ axis as

\section{(c) The Author(s) 2019}

(c) (i) Open Access This article is licensed under a Creative Commons Attribution 4.0 International License, which permits use, sharing, adaptation, distribution and reproduction cc) in any medium or format, as long as you give appropriate credit to the original author(s) and the source, provide a link to the Creative Commons license, and indicate if changes were made. The images or other third party material in this article are included in the article's Creative Commons license, unless indicated otherwise in a credit line to the material. If material is not included in the article's Creative Commons license and your intended use is not permitted by statutory regulation or exceeds the permitted use, you will need to obtain permission directly from the copyright holder. To view a copy of this license, visit http://creativecommons.org/licenses/by/4.0/. 
a crucial switch regulating the proliferation and migration of HCC cells.

\section{Methods}

\section{Cell lines, cell culture, and cell transfection}

The HCC cell lines HepG2 and MHCC-LM3 were obtained from the American Type Culture Collection (ATCC, Manassas, VA, USA) and China Center for Type Culture Collection (CCTCC, China) and cultured in Dulbecco's Modified Eagle's medium (DMEM) supplemented with $10 \%(\mathrm{v} / \mathrm{v})$ fetal bovine serum (FBS) at a humidified incubator with $5 \% \mathrm{CO}_{2}$ at $37^{\circ} \mathrm{C}$.

HBx and CREB1 overexpression was achieved by transfection of $\mathrm{HBx}$ (Plasmid \#42596, Addgene, MA, USA) or CREB1-overexpressing vectors (GeneCopoecia, Guangzhou, China). The empty vector pcDNA3.1 (vector NC) was used as control; CTTN knockdown was achieved by transfection of small interfering RNAs targeting CTTN (si-CTTN; GeneCopoeia, Maryland, USA). HCC cells $\left(1.5 \times 10^{5}\right)$ grown on six-well plates were transfected with $100 \mathrm{pmol}$ siRNA or $2 \mu \mathrm{g}$ of overexpression vectors using $6 \mu \mathrm{L}$ of Lipofectamine 2000 (\#11668019, Invitrogen, MA, USA) as described by the manufacturer. The cells were harvested after $48 \mathrm{~h}$. Western blot analyses or other experiments were performed. The siRNA and vector construction primer sequences are listed in Table S1.

\section{Establishment of stably HBx-expressing HepG2 cells}

Target HBx sequences of pcDNA3.1-Flag HBx plasmid (Plasmid \#42596, Addgene, MA, USA) were amplified by PrimeSTAR GXL DNA polymerase (\#R050A, Takara, Dalian, Japan). Next, HBx gene products and pLV-cDNA (No. 632177, Clontech, CA, USA) were amplified via enzyme digestion and purification according to the manufacturer's instructions. After the enzyme-linked reaction, amplification, and DNA sequencing, we constructed a recombinant $\mathrm{pLV}$-cDNA-HBx plasmid through the recombination of the digested $\mathrm{HBx}$ fragment and the purified pLV-cDNA product. According to the lentiviral packaging system protocol (Clontech, CA, USA), we collected lentiviral particles by transient transfection of $293 \mathrm{~T}$ cells, and then infected HepG2 cells with lentivirus. A Blasticidin S (\#60218ES10, Yeasen, Shanghai, China) concentration of $6 \mu \mathrm{g} / \mathrm{mL}$ selected stably expressing $H B x$ target cells. In this study, the stably modified cells ( $\mathrm{HBx}-$ HepG2) were only used in the identification of proteins interacting with $\mathrm{HBx}$, verification of the interaction between CTTN and HBx, and determination of CTTN mRNA levels and CTTN protein stability experiments.

\section{Immunoprecipitations}

Stably HBx-expressing HepG2 cells were lysed in a coimmunoprecipitation lysis buffer (buffer composed of
$20 \mathrm{mM}$ Tris (pH 7.5), $150 \mathrm{mM} \mathrm{NaCl}$, and $1 \%$ Triton $\mathrm{X}$ 100; P0013, BBI, Shanghai, China) containing a protease inhibitor cocktail for $60 \mathrm{~min}$ at $4{ }^{\circ} \mathrm{C}$. After centrifugation, the supernatant was incubated with anti-mouse IgG (\#3420, CST, MA, USA) at $4{ }^{\circ} \mathrm{C}$ for $60 \mathrm{~min}$ and then with $20 \mu \mathrm{L}$ of protein $\mathrm{G}$ agarose beads (\#37478, CST, MA, USA); an anti-HBx antibody (M10514, Xiamen Innovax Biotech, Xiamen, China) was then added, and the mixture was incubated at $4{ }^{\circ} \mathrm{C}$ overnight. Protein complexes containing the $\mathrm{HBx}$ antibody were precipitated with antimouse IgG beads, and washed with RIPA lysis buffer (P0013, BBI, Shanghai, China) and phosphate-buffered saline (PBS), sequentially. Finally, the pellet was eluted and subjected to SDS-PAGE analysis.

\section{Mass spectrometry (MS) and database analysis}

LC-ESI-LTQ-Orbitrap-MS analysis of proteins was performed as previously described by $\mathrm{us}^{29}$. The protein bands were excised and destained with $100 \mathrm{mM}$ $\mathrm{NH}_{4} \mathrm{HCO}_{3}$ in $50 \%$ acetonitrile $(\mathrm{ACN})$. The proteins were reduced $\left(10 \mathrm{mM}\right.$ dithiothreitol, $\left.56^{\circ} \mathrm{C}, 30 \mathrm{~min}\right)$, alkylated (50 $\mathrm{mM}$ iodoacetamide, in the dark, $20-25^{\circ} \mathrm{C}, 30 \mathrm{~min}$ ), and dried in a vacuum centrifuge. The gel pieces that contained proteins were incubated in a digestion solution (40 mM NH $\mathrm{HCO}_{3}, 9 \% \mathrm{ACN}$, and $20 \mu \mathrm{g} / \mathrm{mL}$ trypsin) at $37^{\circ} \mathrm{C}$ for $18-24 \mathrm{~h}$. The tryptic peptide mixture was purified with a ZipTipC18 microcolumn (cat. no. ZTC18S096; Millipore, Darmstadt, Germany). The purified tryptic peptide mixture was separated onto a PepMap C18 trap column $(75 \mu \mathrm{m}, 15 \mathrm{~cm})$ at a column flow rate of $200 \mathrm{~nL} / \mathrm{min}$. The MS scan and MS spectra were measured in data-dependent mode with MS/MS analysis of the seven strongest ions in the LTQ. The MS data were analyzed using the Xcalibur software and submitted to the database search via Proteome Discoverer. Search parameters included taxonomy (Homo sapiens), enzyme (trypsin), MS/MS tolerance $( \pm 0.5 \mathrm{Da})$, peptide tolerance (20 ppm), peptide charge $(2+, 3+$, and $4+)$, missed cleavage sites (2), fixed modification (carbamoyl methylation of cysteine), and variable modification (methionine oxidation).

\section{Western blot analysis}

Cells were lysed in RIPA lysis buffer (BBI) and separated by electrophoresis, transferred to membranes, and subjected to western blot according to the standard procedure. The primary antibodies used included anti- $\mathrm{HBx}$ (dilution 1:1000, M10514, Xiamen Innovax Biotech, Xiamen, China), anti-CTTN (dilution 1:1000, ab81208, Abcam), anti-CREB1 (dilution 1:1000, Cat. \#9197, CST), anti-cyclin D1 (dilution 1:1000, D220509, BBI), antiMMP-9 (dilution 1:1000, ab73734, Abcam), anti-GAPDH (dilution 1:2000, ab8245, Abcam), anti-E-cadherin (Cat. 
20874, Proteintech), and anti-vimentin (dilution 1:1000, sc-80975, Santa Cruz). After incubating with goat antimouse and goat anti-rabbit secondary antibodies for $1 \mathrm{~h}$ at room temperature, immunoreactive bands were visualized with a chemiluminescence system and quantified using Image J software.

\section{Immunofluorescence staining}

For the detection of the intracellular distribution of $\mathrm{HBx}$ and CTTN, cells $\left(1 \times 10^{5}\right.$ per well $)$ were seeded in six-well glass-bottom plates, fixed in $4 \%$ paraformaldehyde for $15 \mathrm{~min}$, and then permeabilized with $0.2 \%$ Triton X-100 (PBS) for $10 \mathrm{~min}$. Nonspecific binding sites were blocked with $1 \%$ bovine serum albumin in PBS for $1 \mathrm{~h}$. Cells were treated with a primary antibody specific for $\mathrm{HBx}(1: 1000$, M10514, Xiamen Innovax Biotech) or CTTN (dilution 1:1000, ab81208, Abcam) overnight at $4{ }^{\circ} \mathrm{C}$. Thereafter, the cells were incubated with eFluor570-F(ab')2-goat antimouse IgG/IgM $(\mathrm{H}+\mathrm{L})$ (dilution 1:20, Cat. \# 41-4010-82, Invitrogen) and FITC-goat anti-Rabbit IgG $(\mathrm{H}+\mathrm{L})$ crossadsorbed secondary antibody (dilution 1:20, Cat. \#F-2765, Invitrogen, Carlsbad, CA, USA). DAPI (Beyotime, Shanghai, China) was used to stain nuclei before capturing images. The images were acquired using a confocal microscope (Zeiss, Oberkochen, Germany). The green fluorescence indicated HBx expression, the red fluorescence indicated CTTN expression, and the blue fluorescence indicated the nuclei.

\section{Protein-protein interaction analysis}

The Mentha (http://mentha.uniroma2.it/about.php), BioGrid (https://thebiogrid.org/), IntAct (https://www. ebi.ac.uk/intact/), and BIND (https://www.bindingdb. org/bind/aboutus.jsp) databases were used to analyze the proteins interacting with HBx or CTTN. The candidate proteins were then applicated for Kyoto Encyclopedia of Genes and Genomes (KEGG) pathway annotation (http://www.genome.jp/kegg/) to identify the related pathways. The interaction network of these proteins was constructed using String (https://string-db. $\mathrm{org} /$ ) and visualized with the Cytoscape software (version 3.4) 18.

\section{MTT assays}

MTT assays were performed to evaluate cell viability. Twenty-four hours after seeding into 96 -well plates $(5 \times$ $10^{3}$ cells/well), HepG2 and MHCC-LM3 cells were transfected with si-CTTN; $48 \mathrm{~h}$ after transfection, $20 \mu \mathrm{L}$ of MTT (at a concentration of $5 \mathrm{mg} / \mathrm{mL}$; Sigma-Aldrich) was added, and the cells were incubated for an additional $4 \mathrm{~h}$ in a humidified incubator with $5 \% \mathrm{CO}_{2}$ at $37^{\circ} \mathrm{C}$. DMSO $(200 \mu \mathrm{L})$ was added after the supernatant was discarded to dissolve the formazan crystals, and finally absorbance at $490 \mathrm{~nm}$ was measured (PerkinElmer). The viability of non-treated cells (control) was defined as $100 \%$, and the viability of the other groups of cells was calculated accordingly.

\section{5-Bromo-2-deoxyuridine assays}

5-Bromo-2-deoxyuridine (BrdU) assays were conducted 24 and $48 \mathrm{~h}$ after cells were co-transfected with si-CTTN and pcDNA3.1 $(+)$-HBx-overexpressing vectors. Cells were seeded in 96-well culture plates at a density of $2 \times$ $10^{3}$ cells/well, cultured for $48 \mathrm{~h}$, and then incubated with a final concentration of $10 \mu \mathrm{M}$ BrdU (BD Pharmingen, San Diego, CA, USA) for $2 \mathrm{~h}$. Cells were fixed for $30 \mathrm{~min}$ at room temperature, incubated with peroxidase-coupled BrdU antibody (Sigma-Aldrich) for $60 \mathrm{~min}$ at room temperature, washed three times with PBS, incubated with peroxidase substrate (tetramethylbenzidine) for $30 \mathrm{~min}$, and the 450-nm absorbance values were measured for each well. Background BrdU immunofluorescence was determined in cells not exposed to BrdU and stained with the BrdU antibody.

\section{Transwell assays}

Cells were co-transfected with si-CTTN and pcDNA3.1 $(+)$-HBx-overexpressing vectors, and seeded on the top side of polycarbonate transwell filters. The cells were suspended in a medium without serum, while the bottom chamber was filled with medium with serum. After $24 \mathrm{~h}$, the non-migrating cells in the top chambers were removed with cotton swabs. The migrated cells on the lower membrane surface were fixed in $100 \%$ methanol for $10 \mathrm{~min}$, air-dried, stained with crystal violet (BBI, Shanghai, China), and counted under a microscope.

\section{Cell cycle analysis}

Cells $\left(5 \times 10^{5}\right)$ were silenced for CTTN or transfected with a plasmid CREB1 knockdown for $48 \mathrm{~h}$. The cells were washed with PBS and collected into a $1.5-\mathrm{mL}$ centrifuge tube. Subsequently, the cells were fixed with $70 \%$ ethanol for $24 \mathrm{~h}$ and then incubated with a propidium iodide solution (C1052, BBI, Shanghai, China). Flow cytometry was used to study the cell cycle distribution after HBx overexpression and CREB1 knockdown, according to the manufacturer's instructions (Millipore Guava easyCyte, ON, Canada).

\section{Quantitative PCR (qPCR) and tissue specimens}

The total RNA was isolated from stably $\mathrm{HBx}$-expressing HepG2 cells and $\mathrm{HBx}(+) /(-)$ HCC tissues by using TRIzol (Invitrogen) following the manufacturer's protocol. A SYBR green PCR Master Mix (Qiagen) was used for mRNA expression detection following the protocol of the manufacturer. The GAPDH expression was used as an endogenous control. The $2^{-\Delta \Delta C T}$ method $^{30}$ was used to calculate the relative fold changes. 


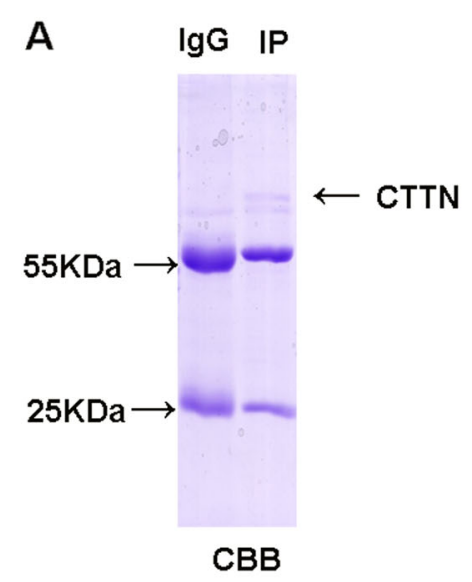

B

D

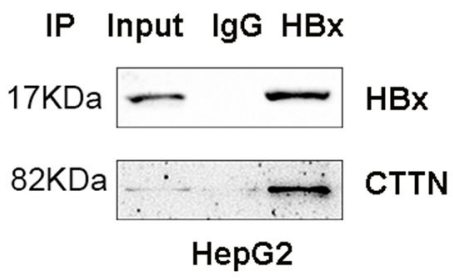

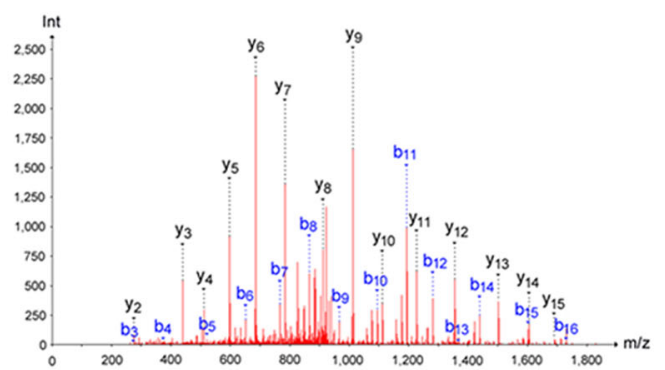

\section{C}

1 MWKASAGHAV SIAQDDAGAD DWETDPDFVN OVSEKEQRWG AKTVQGSGHO

51 EHINIHKLRE NVFQEHQTLK EKELETGPKA SHGYGGKFGV EQDRMDKSAV

101 GHEYQSKLSK HCSQVDSVRG FGGKFGVOMD RVDQSAVGFE YOGKTEKHAS

151 QKDYSSGFGG KYGVQADRVD KSAVGFDYQG KTEKHESQRD YSKGFGGKYG

201 IDKDKVDKSA VGFEYQGKTE KHESQKDYVK GFGGKFGVQT DRQDKCALGW

251 DHQEKLQLHE SQKDYKTGFG GKFGVQSERQ DSAAVGFDYK EKLAKHESQQ

301 DYSKGFGGKY GVQKDRMDKN ASTFEDVTOV SSAYOKTVPV EAVTSKTSNI

351 RANFENLAKE KEQEDRRKAE AERAQRMAKE RQEQEEARRK LEEQARAKTQ

401 TPPVSPAPQP TEERLPSSPV YEDAASFKAE LSYRGPVSGT EPEPVYSMEA

451 ADYREASSQQ GLAYATEAVY ESAEAPGHYP AEOSTYDEYE NDLGITAVAL

501 YDYQAAGDDE ISFDPDDIIT NIEMIDDGWW RGVCKGRYGL FPANYVELRQ
E $\quad H B x$

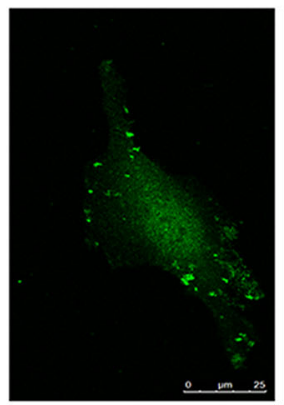

CTTN

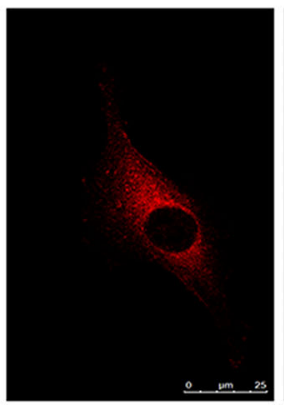

Merge

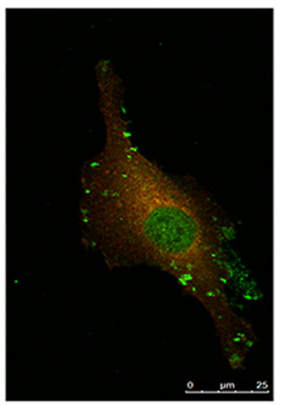

HBx-HepG2

Fig. 1 CTTN interacts with HBx in HepG2 HCC cells. a Analysis of HBx-associated proteins by ColP-MS in HepG2 cells overexpressing HBx (HBXHepG2). $\mathbf{b}$ Representative MS/MS spectrum of CTTN; the amino acid sequence of a doubly charged peptide with m/z 937.9402 was identified as NASTFEDVTQVSSAYQK from mass differences in the $y$ and $b$ fragment ions series. $\mathbf{c}$ Protein sequence of CTTN: the matched peptides are labeled in red bold letters. $\mathbf{d}$ CoIP to validate the HBx-CTTN interaction. CTTN was immunoprecipitated by an anti-HBx but not by lgG control. e Expression of $\mathrm{HBx}$ and CTTN in the cytoplasm HBx-HepG2 by confocal microscopy
Thirty HBx $(+)$ and $22 \mathrm{HBx}(-)$ HCC tissues were collected from tumor surgical resection in the Xiangya Hospital (Central South University, Changsha, China) with the approval of the Medical Ethics Committee of Xiangya hospital at Central South University. Informed consent was obtained from all patients enrolled.

\section{Statistical analysis}

The data were processed using SPSS 17.0 statistical software and presented as the mean \pm SD of at least three independent experiments. A Student's $t$ test was used for statistical comparison between means, where applicable. Differences between more than two groups were estimated using one-way ANOVA. ${ }^{*} P<0.05$; ${ }^{* *} P<0.01$.

\section{Results}

Identification of proteins interacting with $\mathrm{HBx}$ in $\mathrm{HepG} 2$ cells

To identify proteins that interact with $\mathrm{HBx}$, we used a CoIP-MS proteomic analysis of the HBx complexes isolated from HepG2 stably expressing HBx (HBx-HepG2) cells using a $\mathrm{HBx}$ antibody; nonimmune IgGs were used 


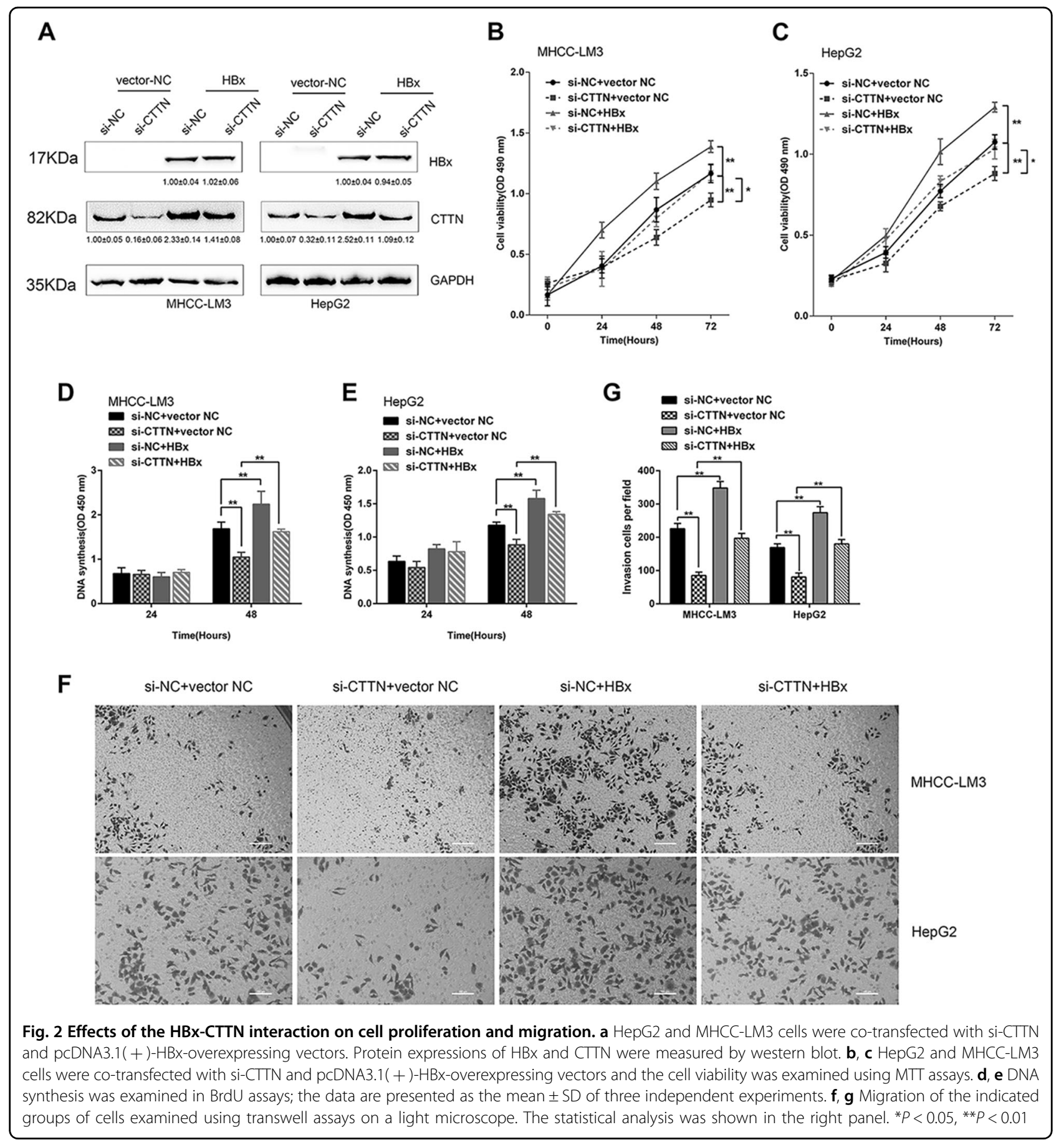

as a negative control. The complexes were eluted and separated on $12 \%$ SDS-PAGE (Fig. 1a). Proteins in the gels were detected by Coomassie brilliant blue staining (CBB), in-gel trypsin digested, and subjected to MS analysis. Fifteen proteins were identified in the anti-HBx-treated cells and not in the negative control: their mass spectra are shown as supplementary data (Table S2). Among them, CTTN was identified from a specific band that originated the highest score and 18 unique peptides. We confirmed the protein sequence of CTTN (Fig. 1b-c; the matched peptides are labeled in red bold letters).

\section{Verification of the interaction between CTTN and $\mathrm{HBx}$}

We confirmed the interaction between CTTN and $\mathrm{HBx}$ in co-immunoprecipitation assays. HBx-HepG2 cell lysates were immunoprecipitated with an $\mathrm{HBx}$ antibody or IgG; immunocomplexes were separated on a gel and subjected to western blot with a CTTN antibody. As 


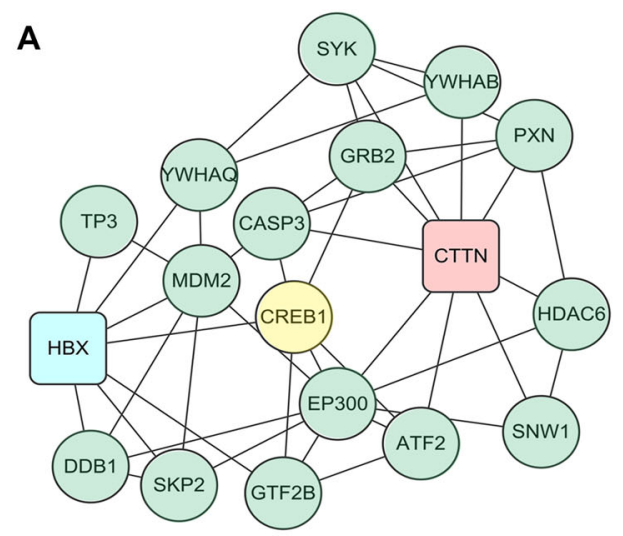

B
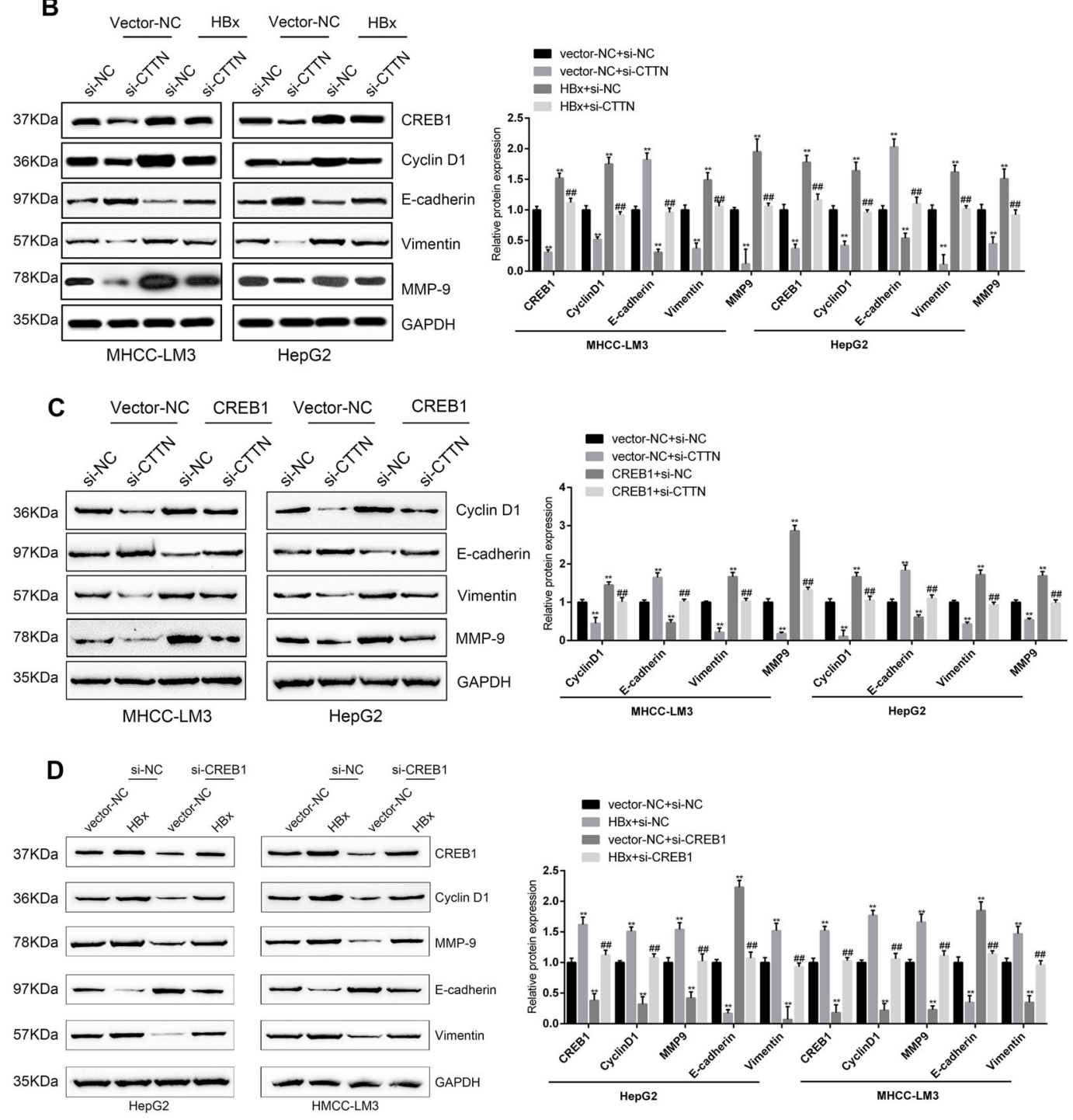

Fig. 3 (See legend on next page.) 
(see figure on previous page)

Fig. 3 The HBx-CTTN interaction modulates CREB1 and its downstream target genes. a Analysis of HBX and CTTN protein-protein network using online databases. The network nodes represent proteins, and the connecting lines represent protein-protein interactions. b CREB1, Cyclin D1, MMP-9, E-cadherin, and vimentin were detected by western blot in HepG2 and MHCC-LM3 cells. HepG2 and MHCC-LM3 cells were transfected with si-CTTN or si-NC and pcDNA3.1 ( + )-HBx or vector NC. si-CTTN or si-NC and pcDNA3.1 ( + )-HBx or vector NC were compared with the si-NC + vectorNC group. ${ }^{*} P<0.01$. si-CTTN and pcDNA3.1 ( + )-HBx group were compared with HBx + si-NC group. ${ }^{\# \#} P<0.01$. c The expression of EMT-associated proteins was detected in HepG2 and MHCC-LM3 cells transfected with si-CTTN or si-NC and CREB1 or vector NC. si-CTTN or si-NC and CREB1 or vector NC were compared with the si-NC + vector-NC group. ${ }^{*} P<0.01$. si-CTTN and the CREB1 group were compared with the CREB1 + si-NC group. ${ }^{\# \#} P<$ 0.01. d The expression of CTTN, HBX, CREB1, cyclin D1, MMP-9, E-cadherin, and vimentin was determined by western blot when HepG2 and MHCCLM3 cells were transfected with si-CREB1 or si-NC and pCDNA3.1 + )-HBx or vector NC compared with the si-NC + vector-NC group. ${ }^{* *} P<0.01$. siCREB1 and pcDNA3.1 ( + )-HBx group were compared with the $\mathrm{HBx}+$ si-NC group. ${ }^{\# \#} P<0.01$

shown in Fig. 1d, CTTN was detected in anti-HBx immunoprecipitates but not in the control samples, suggesting the interaction between CTTN and $\mathrm{HBx}$ in vitro. Subsequently, we investigated CTTN and HBx subcellular localization in HBx-HepG2 cells to determine where they interacted with each other. Immunofluorescence staining indicated that HBx enhanced CTTN fluorescence intensity (Fig. 1d; Supplementary Fig. S1). Moreover, CTTN and HBx colocalized in the cytoplasm (Fig. 1e).

\section{Roles of $\mathrm{HBx} / \mathrm{CTTN}$ interaction in enhancing cell proliferation and migration}

To investigate whether the HBx/CTTN interaction had an impact on cellular processes, we conducted MTT, $\mathrm{BrdU}$, and transwell assays. We found that HBx significantly increased the CTTN in HepG2 and MHCCLM3 cells. While CTTN silencing could partially reverse the effect of HBx on CTTN protein expression (Fig. 2a). The upregulation of CTTN expression by HBx might be associated with the increase of protein stability (Supplementary Fig. S2). MTT and BrdU assays showed that the viability and DNA synthesis ability of HepG2 and MHCCLM3 cells increased upon $\mathrm{HBx}$ overexpression and decreased upon CTTN silencing, and CTTN silencing reversed the effects of $\mathrm{HBx}$ overexpression in cells that overexpressed HBx and were silenced for CTTN (Fig. 2b-e). Similarly, transwell assays revealed that cell migration was significantly suppressed by CTTN knockdown and promoted by HBx overexpression (Fig. 2f, g). These findings indicate that $\mathrm{HBx}$ promotes cell proliferation and migration of HCC cells by interacting with CTTN.

\section{HBX-CTTN interaction modulates CREB1 and its downstream target genes}

To investigate how HBx-CTTN modulates cell proliferation and migration, we performed bioinformatics analyses and analyzed the signaling pathways that are involved (Fig. S3). Proteins that might be related to $\mathrm{HBx}$ and CTTN were analyzed using online databases. A total of 124 human proteins were predicted to interact with
CTTN (Table S3), and a total of 20 proteins were predicted to bind to HBx (Table S4). KEGG pathway annotation (Tables S5 and S6) showed that 16 proteins (nine for CTTN and seven for HBx) were involved in viral carcinogenesis (hsa05023); these proteins were selected and visualized using Cytoscape. We found that, among them (Fig. 3a), a cellular transcription factor was reported to participate in $\mathrm{HBx}$-induced dysregulation of lipogenesis in HCC cells ${ }^{31}$. Previous studies have shown that CREB1 knockdown inhibits the expression of its downstream targets: cyclin D1, Bcl-2, and MMP-9 in gastric cancer ${ }^{2}$. Based on its essential role in $\mathrm{HCC}$, CREB1 was chosen as a potential target. Therefore, we decided to focus on CREB1 as the possible target of HBx-CTTN.

To evaluate the combined effects of HBx and CTTN on CREB1 and its downstream target genes, MHCC-LM3 and HepG2 cells were silenced for CTTN, and the protein levels of CREB1, cyclin D1, E-cadherin, vimentin, and MMP-9 were examined. We found that CTTN knockdown remarkably increased E-cadherin protein levels and reduced the levels of CREB1, cyclin D1, vimentin, and MMP-9, while HBx overexpression decreased E-cadherin expression and increased the levels of CREB1, cyclin D1, vimentin, and MMP-9, and the effect of HBx overexpression was partially attenuated by CTTN knockdown (Fig. 3b). The epithelial-mesenchymal transition (EMT) is directly linked to tumor initiation and invasion ${ }^{32}$. Therefore, we monitored the expression of EMT markers and found that CREB1 overexpression significantly reduced E-cadherin expression and increased vimentin and MMP-9 levels in MHCC-LM3 and HepG2 cells, and this effect was reversed by CTTN knockdown (Fig. 3c). We further assessed the relationship between $\mathrm{HBx}-\mathrm{CTTN}$ and CREB1. The western blot results showed that CREB1 knockdown increased E-cadherin expression and reduced MMP-9 and vimentin expression;these effects were reversed by HBx overexpression (Fig. 3d). We found that CREB1 knockdown inhibited cell migration and induced cell cycle arrest at G1, while these effects could be reversed by $\mathrm{HBx}$ overexpression (Fig. $4 \mathrm{a}-\mathrm{c}$ ). Therefore, 


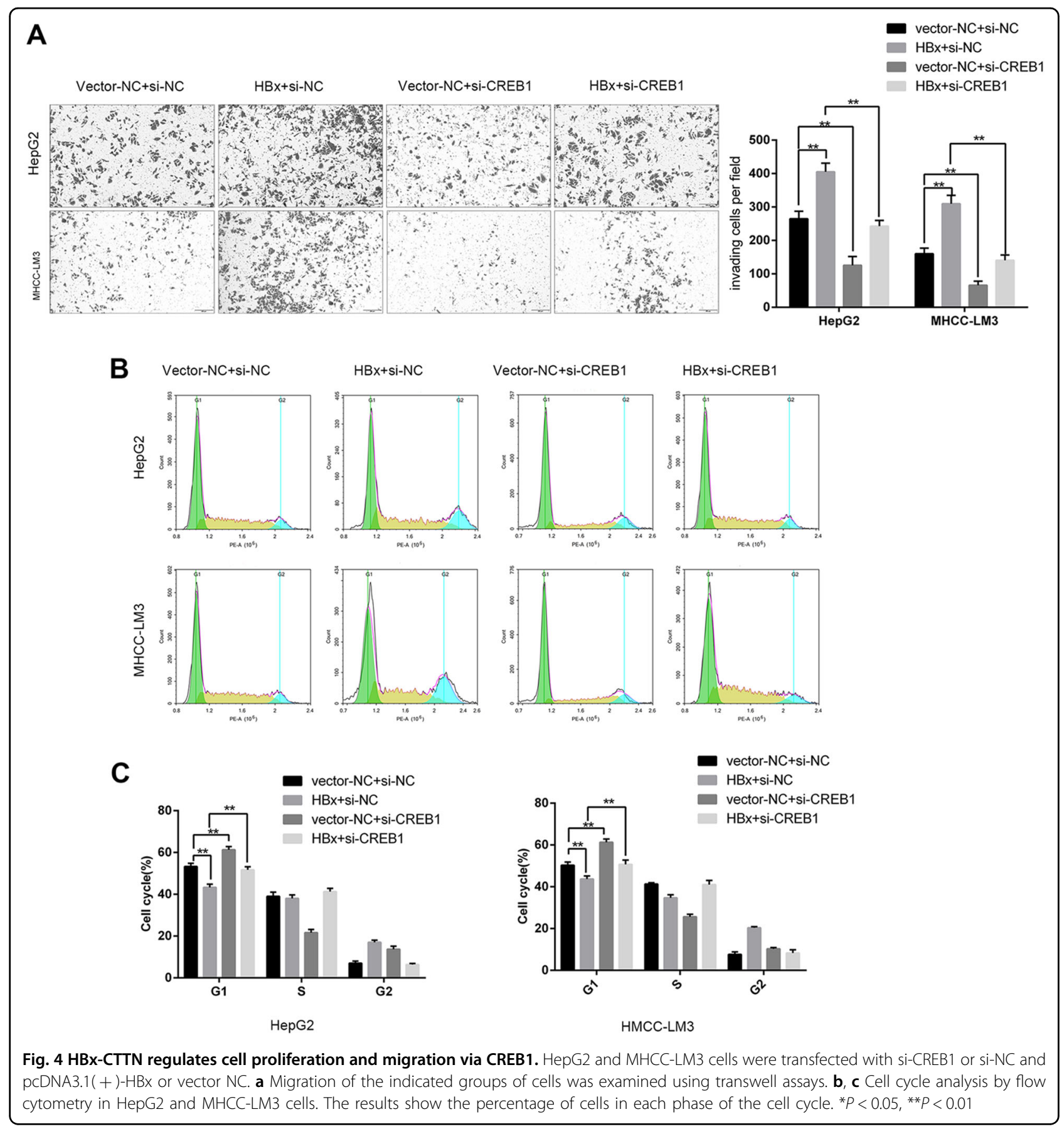

our results suggested that CREB1 and its downstream targets can be regulated by the HBx-CTTN complex.

\section{Positive correlation between the high expression of CTTN and CREB1 in HBV-associated patients}

To investigate the clinical significance of our findings, the mRNA levels of CTTN and CREB1 were evaluated by qPCR in HCC tissues of patients. As shown in Fig. 5, the levels of CTTN in the $\mathrm{HBx}(+)$ group were significantly upregulated compared with the $\operatorname{HBx}(-)$ group $(P<0.01)$, and similar results were obtained with CREB1 $(P<0.01)$. In addition, $C T T N$ and $C R E B 1$ expression were positively correlated in tissue samples.

\section{Discussion}

$\mathrm{HBx}$ viral protein contributes to the development of $\mathrm{HCC}$ mainly through $\mathrm{HBx}$-host interactions, and it is involved in numerous cellular activities, including 
A

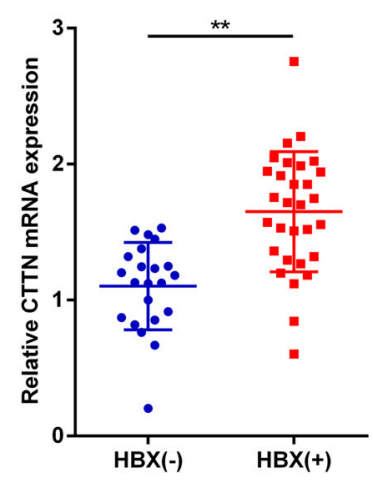

B

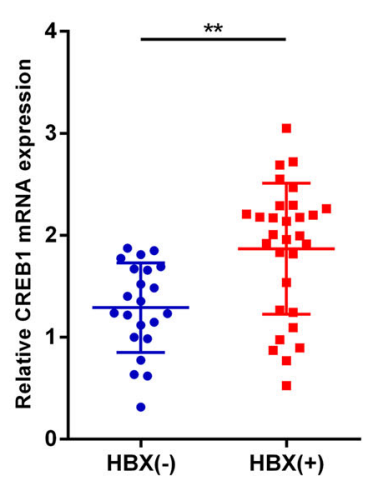

C

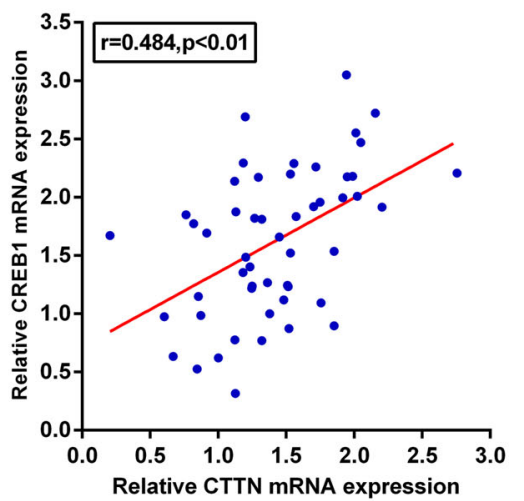

Fig. 5 Upregulation of CTTN and CREB1 in clinical specimens. a, b CTTN and CREB1 expression in $30 \mathrm{HBx}(+)$ and $22 \mathrm{HBx}(-) \mathrm{HCC}$ tissues from patients, as assessed by qPCR. The data are presented as mean \pm SD of three independent experiments. ${ }^{*} P<0.01$. c The correlation between $C T T N$ and CREB1 expression in tissue samples was analyzed using Spearman's rank correlation analysis

transcription activation, transactivation, DNA damage repair, and cell transformation inhibition ${ }^{7,9,13,23,33}$. For example, $\mathrm{HBx}$ inhibits DNA repair by interacting with PARP1 and SIRT6, resulting in dissociation of SIRT6 and PPAP $1{ }^{9}$. HBx also inhibits cell apoptosis by regulating the endoplasmic reticulum (ER) stress response: GRP78 interacts with $\mathrm{HBx}$ in the $\mathrm{ER}$, and this interaction leads to the interruption of ER stress response and host cell apoptosis ${ }^{17}$. Many studies have shown that HBx participates in the regulation of viral or host proteins by protein-protein interactions, providing potent evidence for HBV-related tumorigenesis. However, how HBx protein induces $\mathrm{HCC}$ is still unclarified. In this study, we undertook a proteomics approach to identify $\mathrm{HBx}$ interacting proteins and found that CTTN, a Src substrate, was a novel $\mathrm{HBx}$-interacting protein.

Previous studies have shown that Src protein and RNA expression levels are significantly upregulated in most $\mathrm{HBV}^{+}-\mathrm{HCC}$ specimens and $H B x$ transgenic mice, and the overexpression of Src promotes cell viability, migration, and cell colony formation ${ }^{34-36}$. The Src substrate CTTN has been found to be overexpressed in various cancers, including human colorectal cancer, esophageal tumors, and non-small-cell lung cancer, and HCC and CTTN overexpression closely correlates with tumor aggressiveness, cell adhesion, and cell motility ${ }^{37-42}$. We demonstrated that $\mathrm{HBx}$ contributed to cell migration and proliferation of HCC by binding CTTN in the cytoplasm and upregulating its expression. It has been reported that the phosphorylation and activation of Src are increased in HCC and result in the phosphorylation of CTTN at $\mathrm{Y} 421^{36,43,44}$. This modification is closely associated with CTTN localization and function through the regulation of its interaction with other host proteins ${ }^{45}$. We believe that the adjacent region between Y421 and the SH3 domain of
CTTN (aa 490-550) in the C terminus contributes to the phosphorylation of CTTN at Y421 upon HBx-CTTN interaction, and affects the structure and biological functions of CTTN. This hypothesis needs further investigation. Furthermore, we found that $\mathrm{HBx}$ regulates CTTN protein expression at the post-transcriptional level, and HBx increases CTTN protein stability (Figure S2). Given that Zhao J. et al. reported that CTTN protein degradation was conducted by ubiquitin-independent proteasome system, we assumed that $\mathrm{HBx}$ could increase the stability of CTTN through attenuation of CTTN's ubiquitination ${ }^{46}$.

We also performed bioinformatics analyses to identify the potential targets of $\mathrm{HBx}$ and CTTN. Based on its essential role in HCC, CREB1 was chosen as a potential target. We hypothesized that the $\mathrm{HBx}-\mathrm{CTTN}$ interaction may affect CREB1 and its downstream targets. CREB1 is part of leucine zipper family of proteins, and its expression is essential for various cell functions, including apoptosis, cell cycle, and DNA repair ${ }^{4-49}$. Once activated, CREB1 regulates its downstream target genes, including the apoptosis suppressor gene $B c l 2$, a metalloproteinase involved in extracellular matrix remodeling, $M M P 9$, and the cell cycle-related genes, cyclin $\mathrm{D} 1, \mathrm{~A} 1$, and $\mathrm{B} 1^{2,50}$. Previous studies have revealed that $\mathrm{HBx}$ can induce CREB-mediated activation of $m i R-3188$ and Notch signaling, resulting in the development of $\mathrm{HBV}$-related $\mathrm{HCC}^{51-53}$. Based on our data, we illustrate a critical oncogenic role of CREB1, which is overexpressed and correlates with $\mathrm{CTTN}$ in $\mathrm{HBV}^{+} \mathrm{HCC}$. Furthermore, by targeting CREB1, the $\mathrm{HBx}-\mathrm{CTTN}$ interaction forms a critical oncogenic axis, which regulates cell proliferation, migration, and oncogenic signaling in HCC. Bioinformatics analysis has shown that SKP2, a vital component of the SCF ubiquitin E3 ligase, is involved in HBV-related 
tumorigenesis ${ }^{54}$. CTTN also plays a role in SKP2 expression $^{55,56}$. Therefore, the HBx-CTTN interaction has a possible effect on SKP2. This hypothesis might be investigated in the future.

Based on this work, we provided a novel mechanism of how $\mathrm{HBx}$ contributes to the progression of $\mathrm{HCC}$ : $\mathrm{HBx}$ interacts with CTTN in the cytoplasm, resulting in the upregulation of CREB1, which promotes the proliferation and migration of HCC. The crosstalk between CTTN and $\mathrm{HBx}$ may also provide a potential therapeutic strategy for HCC therapy.

\section{Acknowledgements}

This work was supported by a grant from International Scientific and Technology Cooperation Program of China (No. 2015DFA31490), grants from National Natural Science Foundation of China (No. 81602572, 81873574, and 81402623), and the Natural Science Foundation of Hunan Province (2017JJ3496 and 2018ZX10732202).

\section{Author details}

${ }^{1}$ Department of Infectious Diseases and Hunan Key Laboratory of Viral Hepatitis, Xiangya Hospital, Central South University, Changsha, China. ${ }^{2}$ Center for Molecular Medicine, Xiangya Hospital, Central South University, Changsha, China. ${ }^{3}$ Department of Surgery, UT Southwestern Medical Center, Dallas, Texas, USA. ${ }^{4}$ Department of Blood Transfusion, Xiangya Hospital, Central South University, Changsha, China. ${ }^{5} \mathrm{NHC}$ Key Laboratory of Cancer Proteomics, XiangYa Hospital, Central South University, Changsha, China

\section{Conflict of interest}

The authors declare that they have no conflict of interest.

\section{Publisher's note}

Springer Nature remains neutral with regard to jurisdictional claims in published maps and institutional affiliations.

Supplementary Information accompanies this paper at (https://doi.org/ 10.1038/s41419-019-1650-x).

Received: 24 November 2018 Revised: 30 April 2019 Accepted: 3 May 2019 Published online: 28 May 2019

\section{References}

1. Maluccio, M. \& Covey, A. Recent progress in understanding, diagnosing, and treating hepatocellular carcinoma. CA: a Cancer Journal for Clinicians 62 , 394-399 (2012).

2. Rao, M., Zhu, Y., Cong, X. \& Li, Q. Knockdown of CREB1 inhibits tumor growth of human gastric cancer in vitro and in vivo. Oncol. Rep. 37, 3361-3368 (2017).

3. Luo, J. et al. MicroRNA-146a-5p Enhances Radiosensitivity in Hepatocellular Carcinoma through Replication Protein A3 Induced Activation of the DNA Repair Pathway. Am. J. Physiol. Cell Physiol. 316, C299-C311 (2018).

4. Chen, R. C. et al. Integrated analysis of microRNA and mRNA expression profiles in HBx-expressing hepatic cells. World J. Gastroenterol. 23, 1787-1795 (2017).

5. Choi, J. et al. Risk of hepatocellular carcinoma in patients treated with entecavir vs tenofovir for chronic hepatitis B: A Korean Nationwide Cohort Study. JAMA Oncol. 5, 30-36 (2018).

6. Fu, S. et al. Detection of HBV DNA and antigens in HBsAg-positive patients with primary hepatocellular carcinoma. Clin. Res. Hepatol. Gas. 41, 415-423 (2017).

7. Duan, M. et al. Diverse modes of clonal evolution in HBV-related hepatocellular carcinoma revealed by single-cell genome sequencing. Cell Res. $\mathbf{2 8}$ 359-373 (2018)
8. Yuan, L. et al. Optimized HepaRG is a suitable cell source to generate the human liver chimeric mouse model for the chronic hepatitis B virus infection. Emerg Microbes Infect 7, 144 (2018).

9. $\mathrm{Na}$, T. Y. et al. Interaction of hepatitis B virus $X$ protein with PARP1 results in inhibition of DNA repair in hepatocellular carcinoma. Oncogene 35 , 5435-5445 (2016).

10. Bai, W. et al. Re-designed recombinant hepatitis B virus vectors enable efficient delivery of versatile cargo genes to hepatocytes with improved safety. Viruses 8, 129 (2016).

11. An, P., Xu, J., Yu, Y. \& Winkler, C. A. Host and viral genetic variation in HBVrelated hepatocellular carcinoma. Front. Genet. 9, 261 (2018).

12. Fu, S. et al. Crosstalk between hepatitis $B$ virus $X$ and high-mobility group box 1 facilitates autophagy in hepatocytes. Mol. Oncol. 12, 322-338 (2018).

13. Chen, S. L. et al. HBX-mediated decrease of AIM2 contributes to hepatocellular carcinoma metastasis. Mol. Oncol. 11, 1225-1240 (2017).

14. Tian, $\mathrm{Y}$. et al. HBx promotes cell proliferation by disturbing the cross-talk between miR-181a and PTEN. Sci. Rep. 7, 40089 (2017).

15. Sitterlin, D. et al. Transy C. Interaction of the UV-damaged DNA-binding protein with hepatitis $B$ virus $X$ protein is conserved among mammalian hepadnaviruses and restricted to transactivation-proficient $X$-insertion mutants. J. Virol. 71, 6194-6199 (1997).

16. Wei, $C$. et al. The hepatitis $B$ virus $X$ protein disrupts innate immunity by downregulating mitochondrial antiviral signaling protein. J. Immunol. 185 1158-1168 (2010).

17. Li, J. et al. Hepatitis B virus $X$ protein inhibits apoptosis by modulating endoplasmic reticulum stress response. Oncotarget 8, 96027-96034 (2017).

18. Hsieh, A., Kim, H. S., Lim, S. O., Yu, D. Y. \& Jung, G. Hepatitis B viral X protein interacts with tumor suppressor adenomatous polyposis coli to activate Wnt/ beta-catenin signaling. Cancer Lett. 300, 162-172 (2011).

19. Abdul, F. et al. Smc5/6 antagonism by $\mathrm{HBx}$ is an evolutionarily conserved function of hepatitis B virus infection in mammals. J Virol. 92, e00769-18 (2018).

20. Choi, B. H., Park, G. T. \& Rho, H. M. Interaction of hepatitis B viral X protein and CCAAT/ enhancer-binding protein alpha synergistically activates the hepatitis B viral enhancer II/pregenomic promoter. J. Biol. Chem. 274, 2858-2865 (1999).

21. Chan, $C$. et al. Global re-wiring of p53 transcription regulation by the hepatitis B virus X protein. Mol. Oncol. 10, 1183-1195 (2016).

22. Shukla, R. et al. Proinflammatory cytokine TNF-alpha increases the stability of hepatitis B virus X protein through NF-kappaB signaling. Carcinogenesis 32 , 978-985 (2011)

23. Jiang, T., Liu, M., Wu, J. \& Shi, Y. Structural and biochemical analysis of BCl-2 interaction with the hepatitis B virus protein HBx. Proc. Natl Acad. Sci. USA 113 2074-2079 (2016)

24. Huang, W. G. et al. Targeted proteomic analysis of 14-3-3sigma in nasopharyngeal carcinoma. Int. J. Biochem. Cell Biol. 42, 137-147 (2010).

25. Zhang, $T$. et al. An integrated proteomics and bioinformatics analyses of hepatitis $B$ virus $X$ interacting proteins and identification of a novel interactor apoA-I. J. proteomics 84, 92-105 (2013).

26. Levrero, M. \& Zucman-Rossi, J. Mechanisms of HBV-induced hepatocellular carcinoma. J. Hepatol. 64(1 Suppl), S84-S101 (2016).

27. Al-Anazi, M. R., Nazir, N., Colak, D., Al-Ahdal, M. N. \& Al-Qahtani, A. A. Deletion and functional analysis of hepatitis B virus $X$ protein: evidence for an effect on cell cycle regulators. Cell. Physiol. Bioch.: Int. J. Exp. Cell. Physiol., Bioch., And Pharmacol. 49, 1987-1998 (2018).

28. Bagga, S., Rawat, S., Ajenjo, M. \& Bouchard, M. J. Hepatitis B virus (HBV X protein-mediated regulation of hepatocyte metabolic pathways affects viral replication. Virology 498, 9-22 (2016).

29. Peng, F. et al. Nitroproteins in human astrocytomas discovered by gel electrophoresis and tandem mass spectrometry. J. Am. Soc. Mass Spectrom. 26, 2062-2076 (2015).

30. Livak, K. J. \& Schmittgen, T. D. Analysis of relative gene expression data using real-time quantitative PCR and the 2(-Delta Delta C(T)) Method. Methods $\mathbf{2 5}$ 402-408 (2001)

31. You, X. et al. Hepatitis B virus $X$ protein upregulates oncogene Rab18 to result in the dysregulation of lipogenesis and proliferation of hepatoma cells. Carcinogenesis 34, 1644-1652 (2013).

32. von Olshausen, G. et al. Hepatitis B virus promotes beta-catenin-signalling and disassembly of adherens junctions in a Src kinase dependent fashion. Oncotarget 9, 33947-33960 (2018). 
33. Mouzannar, K. et al. Farnesoid $X$ receptor-alpha is a proviral host factor for hepatitis $B$ virus that is inhibited by ligands in vitro and in vivo. FASEB J. $\mathbf{3 3}$ 2472-2483 (2018).

34. Rothschild, B. L. et al. Cortactin overexpression regulates actin-related protein $2 / 3$ complex activity, motility, and invasion in carcinomas with chromosome 11 q13 amplification. Cancer Res. 66, 8017-8025 (2006).

35. MacGrath, S. M. \& Koleske, A. J. Cortactin in cell migration and cancer at a glance. J. Cell Sci. 125(Pt 7), 1621-1626 (2012).

36. Lu, J. W. et al. Identification of the common regulators for hepatocellular carcinoma induced by hepatitis B virus $X$ antigen in a mouse model. Carcinogenesis 33, 209-219 (2012).

37. Hong, C. C. et al. miR326 maturation is crucial for VEGF-C-driven cortactin expression and esophageal cancer progression. Cancer Res. 74, 6280-6290 (2014).

38. Jing, $\mathrm{X}$. et al. Cortactin promotes cell migration and invasion through upregulation of the dedicator of cytokinesis 1 expression in human colorectal cancer. Oncology Rep. 36, 1946-1952 (2016).

39. Li, Y. et al. miR-182 suppresses invadopodia formation and metastasis in nonsmall cell lung cancer by targeting cortactin gene. J. Exp. Clin. Cancer Res. 37, 141 (2018).

40. Jia, D. et al. Amplification of MPZL1/PZR promotes tumor cell migration through Src-mediated phosphorylation of cortactin in hepatocellular carcinoma. Cell Res. 24, 204-217 (2014).

41. Chuma, M. et al. Overexpression of cortactin is involved in motility and metastasis of hepatocellular carcinoma. J. Hepatol. 41, 629-636 (2004).

42. He, J. et al. Fra-1 is upregulated in gastric cancer tissues and affects the PI3K Akt and p53 signaling pathway in gastric cancer. Int. J. Oncol. 47, 1725-1734 (2015).

43. Martinez-Quiles, N., Ho, H. Y., Kirschner, M. W., Ramesh, N. \& Geha, R. S. Erk/Src phosphorylation of cortactin acts as a switch on-switch off mechanism that controls its ability to activate N-WASP. Mol. Cell. Biol. 24, 5269-5280 (2004).

44. Chellaiah, M. A. \& Schaller, M. D. Activation of Src kinase by protein-tyrosine phosphatase-PEST in osteoclasts: comparative analysis of the effects of bisphosphonate and protein-tyrosine phosphatase inhibitor on Src activation in vitro. J. Cell. Physiol. 220, 382-393 (2009).
45. Martini, V., Gattazzo, C. \& Frezzato, F. Cortactin, a Lyn substrate, is a checkpoint molecule at the intersection of BCR and CXCR4 signalling pathway in chronic lymphocytic leukaemia cells. Br J Haematol. 178, 81-93 (2017).

46. Zhao, J. et al. Extracellular signal-regulated kinase (ERK) regulates cortactin ubiquitination and degradation in lung epithelial cells. J. Biol. Chem. 287, 19105-19114 (2012).

47. Mukherjee, S. et al. CDK5 inhibition resolves PKAVCAMP-independent activation of CREB1 signaling in glioma stem cells. Cell Rep. 23, 1651-1664 (2018).

48. Zhang, $P$. et al. Knockdown of CREB1 promotes apoptosis and decreases estradiol synthesis in mouse granulosa cells. Biomed. Pharmacother. 105 1141-1146 (2018)

49. Walia, M. K., Taylor, S., Ho, P. W. M., Martin, T. J. \& Walkley, C. R. Tolerance to sustained activation of the CAMP/Creb pathway activity in osteoblastic cells is enabled by loss ofp53. Cell Death Dis. 9, 844 (2018).

50. Esparza, S. D. et al. CREB regulates Meis1 expression in normal and malignant hematopoietic cells. Leukemia 22, 665-667 (2008).

51. Zhou, S. J., Deng, Y. L., Liang, H. F., Jaoude, J. C. \& Liu, F. Y. Hepatitis B virus X protein promotes CREB-mediated activation of miR-3188 and Notch signaling in hepatocellular carcinoma. Cell Death Differ. 24, 1577-1587 (2017).

52. Liu, F. Y. et al. MiR-216b is involved in pathogenesis and progression of hepatocellular carcinoma through HBx-miR-216b-IGF2BP2 signaling pathway. Cell Death \& Dis. 6, e1670 (2015).

53. Gao, J. et al. Hepatitis B virus $X$ protein activates Notch signaling by its effects on Notch1 and Notch4 in human hepatocellular carcinoma. Int. J. Oncol. 48, 329-337 (2016).

54. Wei, X. et al. SKP2 promotes hepatocellular carcinoma progression through nuclear AMPK-SKP2-CARM1 signaling transcriptionally regulating nutrientdeprived autophagy induction. Cell. Physiol. Biochem.: Int. J. Exp. Cell. Physiol., Biochem., Pharmacol. 47, 2484-2497 (2018).

55. Croucher, D. R., Rickwood, D., Tactacan, C. M., Musgrove, E. A. \& Daly, R. J. Cortactin modulates RhoA activation and expression of Cip/Kip cyclindependent kinase inhibitors to promote cell cycle progression in 11q13amplified head and neck squamous cell carcinoma cells. Mol. Cell. Biol. 30, 5057-5070 (2010)

56. Lee, S., Kim, W., Ko, C. \& Ryu, W. S. Hepatitis B virus X protein enhances Myc stability by inhibiting SCF(Skp2) ubiquitin E3 ligase-mediated Myc ubiquitination and contributes to oncogenesis. Oncogene 35, 1857-1867 (2016). 